\title{
Epidemiology of Children's Individual Sports Injuries
}

\section{An Important Area of Medicine and Sport \\ Science Research}

\author{
Dennis Caine ${ }^{\mathrm{a}}$, Nicola Maffulli ${ }^{\mathrm{b}}$ \\ aDepartment of Physical Education, Health and Recreation, Western Washington \\ University, Bellingham, Wash., USA; 'Keele University School of Medicine, \\ University Hospital of North Staffordshire and Hartshill Orthopedic Surgical Unit, \\ Stoke on Trent, Staffordshire, England
}

\section{Introduction}

Participation in children's and youth sports is increasingly popular and widespread in Western culture. Many of these youngsters initiate year-round training and specialization in their sports at a very early age. This is probably due to the 'catch them young' philosophy, and to the belief that, to achieve international standing in later sporting life, intensive training should be started before puberty [1]. It is not uncommon today, for example, for preteens to train 20 or more hours each week at regional training centers in tennis or gymnastics, to compete in triathlons, or for youngsters as young as 6-8 years of age to play organized hockey or soccer and travel with select teams to other towns and communities to compete against other teams of similar caliber.

\section{The Uniqueness of the Young Athlete}

Engaging in sports activities at a young age has numerous health benefits but also involves risk of injury. Indeed, the young athlete may be particularly vulnerable to sport injury due to the physical and physiological processes of growth. Injury risk factors that are unique to the young athlete include susceptibility to growth plate injury, nonlinearity of growth, limited thermoregulatory 
capacity, and maturity-associated variation. Although problems do not ordinarily arise at normal levels of activity, the more frequent and intense training and competition of young athletes today may create conditions under which this susceptibility exerts itself.

\section{Susceptibility to Growth Plate Injury}

Growth plate injuries have no counterpart in adult life. The fear is that the tolerance limits of the growth plate may be exceeded by the mechanical stress of injury in sports like wrestling or by the repetitive physical loading demanded in gymnastics [2]. The major concern with physeal injuries is that they can produce permanent injury to the growth cells, resulting in growth disturbance.

The resistance of growth plate cartilage to stress is low [3]. It is also less resistant than articular cartilage to compression and shearing, and than adjacent bone to shear and tension forces. In addition, the growth plate may be two to five times weaker than surrounding fibrous tissue [4]. Therefore, when disruptive forces are applied to an extremity, failure may occur through the growth plate. The susceptibility of the growth plate to injury appears to be especially pronounced during periods of rapid growth [5-15]. Research pertaining to the development of physeal cartilage in animals shows a decrease in physeal strength during pubescence [6]. The data on humans are consistent with these findings [7-9]. An increase in the rate of growth at the growth plate is accompanied by structural changes that result in a thicker and more fragile plate [7, 10]. In addition, bone mineralization may lag behind bone linear growth during the pubescent growth spurt, thus rendering the bone temporarily more porous and more subject to injury [11,12]. Studies of the incidence of physeal injuries in humans indicate an increased occurrence of fractures during pubescence [11-15].

Unfortunately, good epidemiological data are lacking on the incidence of physeal injuries in individual sports. However, literature reviews on this topic reveal multiple published case reports and case series that attest to the occurrence of both acute and chronic growth plate injuries in children's individual sports $[2,16,17]$. Reports of sport-related physeal injuries resulting in growth disturbance are also reviewed in these papers $[2,16,17]$.

\section{Nonlinearity of Growth}

The normal growth pattern is nonlinear; that is, differential growth of the body segments (head, trunk, and lower extremities) occurs throughout growth and influences body proportions accordingly [18]. At birth, the relative contribution of head and trunk to total stature is highest and declines through childhood into adolescence. Thus, the child is characterized by a proportionately larger head and trunk, and shorter legs compared to an adult. Consequently, it could be argued that under a given physical load a child's locomotor apparatus is exposed 
to greater stress: hence to a higher risk of overuse injury than that of an adult [16]. Yet, often these child athletes progress rapidly to training regimens, skills, and stunts that were originally introduced and intended for more mature individuals. Indeed, they may well have surpassed the extent of physical loading and skill complexity that characterized training and competition a generation ago.

\section{Limited Thermoregulatory Capacity}

Exercising children do not adapt as effectively as adults when exposed to high temperature. This may affect their performance and wellbeing, and increase the risk for heat-related illness. The thermoregulatory shortcomings of children relative to adults during heat and exercise have recently been reviewed [18].

- children gain heat faster from the environment by convection, conduction, and radiation than do adults as a result of their greater surface area-tobody mass ratio than adults;

- children also produce more metabolic heat per mass unit than adults during activities that include walking and running;

- $\quad$ sweating capacity is considerably lower in children than in adults, which reduces their ability to dissipate body heat by evaporation; and

- children acclimate to exercise in hot weather at a slower rate than do adults.

Thus, a child will generate more heat for a given activity, yet is less able to dissipate body heat particularly in a hot environment. As children frequently do not feel the urge to drink enough to replenish fluid loss either before or following exercise, they may experience dehydration and increased risk of heat illness [19].

\section{Maturity-Associated Variation}

Children of the same chronological age may vary considerably in biological maturity status, and individual differences in maturity status influence measures of growth and performance during childhood and adolescence [18]. For example, the structural, functional, and performance advantages of early-maturity boys in sports requiring size, strength, and power are well known. The fear is that an unbalanced competition between early and late-maturing boys in contact sports such as martial arts and wrestling contributes to at least some of the serious injuries in these sports. A noninvasive method for estimating maturity status as a basis for grouping young athletes has recently been proposed [20]. However, the classification for participation in youth sports continues to rely primarily on chronological age, which may add yet another dimension of individual variation. For example, within a single age group (e.g., 12 years of age), the child who is 12.9 years of age is likely taller, heavier and stronger than the child who is 12.0 years of age, even though both are classified as 12 years of age. Thus, when children are grouped by age, variation is associated with chronological age per se and also with differences in biological maturity [18]. 


\section{Concern for the Health and Safety of Young Athletes}

The increased sports involvement of children from an early age and continued through the years of growth, against a background of their apparent vulnerability to injury, gives rise to concern about the risk and severity and long-term effects of injury. Some recent data suggest that the risk of pediatric sports injury is high and constitutes a significant public health burden. In the United States during 2000-2001, for example, there were an estimated 4.3 million nonfatal sports- and recreation-related injuries treated in US hospital emergency departments [21]. Injury rates for both sexes peaked during the periadolescent years and were highest for boys. Children aged 5-14 years accounted for nearly $40 \%$ of all sports-related injuries [22]. Since only the more serious injuries present to hospital emergency departments, these data reflect only part of the overall injury picture in children's and youth sports. Many more, albeit less severe, injuries are treated in other settings such as healthcare providers' offices and clinics.

Parents need to know about the risks of injuries in children's and youth sports and what they can do to help prevent injury [22]. Indeed, young athletes of all ages and everyone who works with them, whether they be parents, sports medicine personnel, sports governing bodies, or coaches, need to know answers to questions such as the following: Is the risk of injury greater in some sport activities, or level of activity, than in others? What types of injuries are most common in a given sport? What is the average time lost from injury and what is the risk of permanent impairment? Are some children prone to sports injury? Are some physical, psychological, or sport-related factors associated with an increased risk of injury? Can injury be prevented and if so, how? How effective are the preventive measures that have been implemented? These are all questions which sports medicine personnel and coaches should be prepared to respond to, and the information should be made readily available to them. Providing this information, at least as far as possible, is an important objective of sports injury epidemiology research.

\section{Epidemiology of Sports Injuries in Children}

Sports injury epidemiology is the study of the distribution and determinants of varying rates of sports injuries for the purpose of identifying and implementing measures to prevent their development and spread [23]. The epidemiologist in sports medicine is concerned with quantifying injury occurrence (how much) with respect to who is affected by injury, where and when injuries occur, and what is their outcome, for the purpose of explaining why and how 
injuries occur and identifying strategies to control and prevent them. The study of the distribution of varying rates of injuries (i.e., who, where, when, what) is referred to as descriptive epidemiology. The study of the determinants of an exhibited distribution of varying rates of injuries (i.e., why and how) and the effectiveness of selected preventive measures is referred to as analytical epidemiology. For a more extensive discussion of the epidemiological approach to sports injuries the reader is referred to Caine et al. [23].

The epidemiology of sports injuries in children and youth is an important area of research that has been largely overlooked in the medicine and sport science literature; it deserves more serious study, particularly with regards to the identification and analysis of risk factors and preventive measures [24]. However, existing epidemiological research on pediatric sports injuries has already resulted in rule changes, equipment standards, improved coaching techniques, and better conditioning of athletes [24]. For example, the prohibition of 'spearing' in football and rules regarding water depth and the racing dive in swimming are examples of how data on deaths and catastrophic injuries can be used to help promote the safety of young athletes. Other preventive measures supported by research include anchoring movable soccer goals to prevent tipping, improved training for high school wrestling coaches and increased awareness of pathogenic weight control in wrestling and gymnastics [25].

\section{Purpose and Organization of This Book}

The benefits of physical activity for children and youth are substantial. However, growth in sports participation has contributed to an increase in pediatric sports-related injuries. In addition to the immediate healthcare costs, these injuries may have long-term consequences on the musculoskeletal system, resulting in limb dysfunction and a subsequent reduction in levels of physical activity [26]. However, half of all organized sports-related injuries among children can be prevented [27].

The purpose of Epidemiology of Pediatric Sports Injuries: Individual Sports is to review comprehensively what is known about the distribution and determinants of injury rates in a variety of individual sports, and to suggest injury prevention measures and guidelines for further research. This book provides the first comprehensive compilation and critical analysis of epidemiological data over children's individual sports: including equestrian, gymnastics, martial arts, skiing and snowboarding, tennis, track and field, and wrestling. The next volume (vol. 49) in Medicine and Sport Science will address the epidemiology of injuries in pediatric team sports. A common, uniform strategy and evidence-based approach to organizing and interpreting the literature is used 
in all chapters. All the sports-specific chapters are laid out with the same basic headings, so that it is easy for the reader to find common information across chapters. Section headings include, besides the Abstracts and Introductions:

- Incidence of Injury

- Injury Characteristics

- Injury Severity

- Injury Risk Factors

- Suggestions for Injury Prevention

- Suggestions for Further Research

In each sports-specific chapter, an epidemiological picture has been systematically developed from the data available in prospective cohort, retrospective cohort, case-control, and cross-sectional studies (i.e., denominator-based designs). From this picture, it became possible to suggest preventive measures which seemed at least reasonable, given the level of evidence available, and to suggest needed areas for further research. A chapter titled 'Injury Prevention and Future Research' that addresses individual and team sports is included at the end of both volumes to provide a more global, across-sport examination of the literature identifying risk factors and prevention strategies for injury in child and adolescent sports.

Sport scientists and healthcare professionals will find Epidemiology of Pediatric Sports Injuries - both Volume 48: Individual Sports and Volume 49: Team Sports - useful in identifying problem areas in which appropriate preventive measures can be initiated to reduce the risk and severity of injuries. They will also want to use these as a resource for research initiatives in the epidemiology of children's sports injuries. Sports administrators and coaches will find these books a thought-provoking reference that spurs discussion and encourages changes in the rules, equipment standards, coaching techniques, and athlete conditioning programs they use. Finally, the books will provide these individuals with current information on the epidemiology of pediatric sports injuries so that they, in turn, can inform parents about the risks of injury in children's sports and how they can help their children avoid or limit these risks.

\section{References}

1 Maffulli N: Children in sport: Questions and controversies; in Maffulli N (ed): Color Atlas and Text of Sports Medicine in Childhood and Adolescence. London, Mosby-Wolfe, 1995, pp 209-229.

2 Micheli LJ: Pediatric and adolescent sports injury: Recent trends; in Pandolf KP (ed): Exercise and Sport Science Reviews. New York, Macmillan, 1986, pp 359-374.

3 Larson RL, McMahon RO: The epiphyses and the childhood athlete. JAMA 1966;196:607-612.

4 Ogden JA: Skeletal Injury in the Child. New York, Springer, 2000.

5 Carter SR, Aldridge MJ: Stress injury of the distal radial growth plate. J Bone Jt Surg Br 1988;70:834-836. 
6 Bright RW, Burstein AH, Elmore SM: Epiphyseal-plate cartilage: A biomechanical and histological analysis of failure modes. J Bone Jt Surg Am 1974;56:688-703.

7 Alexander CJ: Effect of growth rate on the strength of the growth plate-shaft function. Skeletal Radiol 1976;1:67-76.

8 Morsher E: Strength and morphology of growth cartilage under hormonal influence of puberty. Reconstru Surg Traumatol 1968;10:1-96.

9 Speer DP, Braun JK: The biomechanical basis of growth plate injuries. Phys Sportsmed 1985;13:72-78.

10 Aldridge MJ: Overuse injuries of the distal radial growth epiphysis; in Hoshizaki BT, Salmela JH, Petiot B (eds): Diagnostics, Treatment and Analysis of Gymnastic Talent. Montreal, Sports Psyche Editions, 1987, pp 25-30.

11 Bailey DA, Wedge JH, McCulloch RG, Martin AD, Bernardson SC: Epidemiology of fractures of the distal end of the radius in children as associated with growth. J Bone Jt Surg Am 1989;71:1225-1231.

12 Bradford DS: Vertebral osteochondrosis (Scheuermann's kyphosis). Clin Orthop 1981;158:83-90.

13 Peterson CA, Peterson HA: Analysis of the incidence of injuries to the epiphyseal growth plate. J Trauma 1972;12:275-281.

14 Benton JW: Epiphyseal fracture in sports. Phys Sportsmed 1982;10:63-71.

15 Caine DJ, Lindner K: Growth plate injury: A threat to young distance runners? Phys Sportsmed 1984;12:118-124.

16 Caine D: Growth plate injury and bone growth: An update. Pediatr Exerc Sci 1990;2:209-229.

17 Caine D: Injury and growth; in Sands WA, Caine DJ, Borms J (eds): Scientific Aspects of Women's Gymnastics. Med Sport Sci. Basel, Karger, 2003, vol 45, pp 46-71.

18 Malina RM, Bouchard C, Bar-Or O: Growth, Maturation, and Physical Activity, ed 2. Champaign, Human Kinetics, 2004, pp 267-273.

19 Walker SM, Casa DJ, Levreault ML, Psathas E, Sparrow SL, Decher DR: Children participating in summer sports camps are chronically dehydrated. Med Sci Sports Exerc 2004;36(suppl 5):180-181.

20 Mirwald RL, Baxter-Jones ADG, Bailey DA, Beunen GP: An assessment of maturity from anthropometric measurements. Med Sci Sports Exerc 2002;34:689-694.

21 Centers for Disease Control and Prevention Morbidity and Mortality Weekly Report: Non-fatal sports- and recreation-related injuries treated in emergency departments, United States, July 2000-June 2001. MMWR Weekly 2002;51:736-740. Available from URL: http://www.cdc.gov/ $\mathrm{mmwr} / \mathrm{preview} / \mathrm{mmwrhtml} / \mathrm{mm} 5133 \mathrm{a} 2 . \mathrm{htm}$ [Accessed on July 31, 2004].

22 National Safe Kids Campaign: Get into the game: A national survey of parents' knowledge, attitudes and self-reported behaviors concerning sports safety. Press release, May 4, 2000. Available from URL: http://www.safekids.org/ [Accessed on July 31, 2004].

23 Caine C, Caine D, Lindner K: The epidemiologic approach; in Caine D, Caine C, Lindner K (eds): Epidemiology of Sports Injuries. Champaign, Human Kinetics, 1996, pp 1-13.

24 Mueller F, Blyth C: Epidemiology of injuries in children. Clin Sports Med 1982;1:343-352.

25 Cantu RC, Mueller FO: Fatalities and catastrophic injuries in high school and college sports, 1982-97. Phys Sportsmed 1999;27:35.

26 Arendt EA: Specific injury locations - Overview; in Garrett WE, Lester GE, McGown J, Kirkendall DT (eds): Women's Health in Sports and Exercise. Rosemont, American Academy of Orthopedic Surgeons, 2001, pp 85-86.

27 National Safe Kids Campaign: Injury Facts. Available from URL: http://www.safekids.org/ [Accessed on July 31, 2004].

Prof. Dennis Caine, $\mathrm{PhD}$

Department of Physical Education, Health and Recreation

Western Washington University

Bellingham, WA 98225-9067 (USA)

Tel. +1 360650 3529, Fax +1 360650 7447, E-Mail Dennis.Caine@wwu.edu 